\title{
Post-Hoc Analysis of Data Received with Nuclear Medical Diagnostic in Bulgarien Patients
}

\author{
KRASIMIRA PRODANOVA \\ Faculty of Applied Mathemdtics \\ and Informatics \\ Technical University of Sofia \\ Sofia, BULGARIA
}

\author{
YORDANKA UZUNOVA \\ University Hospital "Lozenets" of \\ Faculty of Medicine \\ Sofia University \\ Sofia, BULGARIA
}

\begin{abstract}
Fever of unknown origin (FUO) is a clinical condition extremely difficult to diagnose. For this purpose a number of methods are used - clinical and paraclinical, as well as medical imaging, of which only nuclear-medical diagnostic methods allow performance of full-body scanning, which avoids skipping areas, loss of time and resources. In this article we are looking for the interrelationship between two groups of factors: group 1 (body temperature; leukocytes and erythrocyte sedimentation rate (ESR) and group 2 (presence and severity of an inflammatory process). Analysis of variance (ANOVA) is used for analyzing observations of the index of accumulation that depends on the effects of the factors as patient's temperature, leukocytes and ESR. The comparison of mean values of the statistically significant factors for the different categories of the index of accumulation and respectively activity of the inflammatory process is made. The study included 74 patients: 30 (40.54\%) men aged 15 to 71 years. and $44(59.46 \%)$ women aged 7 to 74 diagnosed in a hospital in Sofia, Bulgaria. However, ANOVA test results don't map out which groups are different from other groups. To determine whether the mean differences between the factors are statistically significant post hoc test is used.
\end{abstract}

Keywords-ANOVA, Post-hoc test, Nuclear Medical Diagnostic

Received: March 9, 2021. Revised: July 23, 2021. Accepted: August 4, 2021. Published: August 20, 2021.

\section{Introduction}

Clinics often receive patients, who have had fever for a long time, without adequate response to ongoing therapy and without an established diagnosis. Petersdorf [1] defines the term "fever of unknown origin" (FUO) as "Fever with a body temperature above $38.3^{\circ} \mathrm{C}$ for more than 3 weeks without a final diagnosis, despite a one-week hospital stay ". According to leading experts, a differential diagnosis should be made between FUO and four groups of diseases: infectious, malignant, autoimmune and mixed. Infections remain the most common cause of FUO [2, 3, 4].

Nuclear medicine diagnostics have many advantages over the other imaging methods. The most important of these advantages being the diagnosis of the earliest functional changes in an organism, before the development of structural-anatomical changes. Nuclear medicine also allows for whole body scanning (without any omissions of body parts in the scan), as well as saving time.

Until now, using nuclear medicine diagnostics, there is some evidence in the Bulgarian scientific literature of a relationship between body temperature, leukocytes, erythrocyte sedimentation rate (ESR) and the presence and severity of the inflammatory process (IP) $[5,6]$, but the results based on a small samples (maximum size 54 patients).

That's why we decided to look for the existence of such a relationship by applying the diagnostic capacity of nuclear medicine based on the data from random sample of 74 patients.

Analysis of variance (ANOVA) is one of the most frequently used statistical methods in medical research [7]. Analysis of variance (ANOVA) is used for analyzing observations of the index of accumulation (in four groups) that depends on the effects of the factors as patient's body temperature, leukocytes and erythrocyte sedimentation rate (ESR). The comparison of mean values of the statistically significant factors for the four categories of the index of accumulation and respectively activity of the inflammatory process will make it possible to apply the adequate treatment targeted at personalized medicine. To determine whether the mean differences between the factors values are statistically significant post hoc test of Kruskal-Wallis is used.

\section{Materials and Methods}

The data are received from a random sample of 74 patients with fever of unknown origin (FUO): 30 (40.54\%) men aged 15 to 71 years, and $44(59.46 \%)$ women aged 7 to 74 , all diagnosed in a hospital of ministry of interior in Sofia. In 52,70\% (39 persons) there was no initial working diagnosis and therefore they were united in a first subgroup - only fever, while 47,30\% (35 persons) had an initial working diagnosis and were grouped into 9 subgroups, respectively: pyelonephritis -8 ( 2 with +6 without a classic clinical presentation (CCP)); post-operative complications 6 ( 4 with +2 without), sepsis -5 ( 2 with +3 without CCP); Crohn's disease -5 (1 with +4 without CCP); abscess - 4 (1 with +3 without $\mathrm{CCP}$ ); bronchopneumonia - 3 without CCP; pleurisy - 2 without CCP; alopecia- 2 without CCP; Lyme disease - 2 without CCP. The objectives of the nuclear medical diagnostic study are:

First: Evaluation of the quality of the ${ }^{99 \mathrm{~m}} \mathrm{Tc}-\mathrm{HMPAO}$ leukocyte tagging technique used. For this purpose, an "efficiency of tagging" (ET) of the leukocytes is used and is equal to: $\left(\mathrm{A}_{\text {sample }}\right) /\left[\mathrm{A}_{\text {sample }}+\mathrm{A}_{\text {aspirated Plasma }}\right]$, where $\mathrm{A}_{\text {sample }}$ is activity of the sample. 
The value proves the quality of the used technique for isolation and labeling of leukocytes, which is a prerequisite for achieving a high quality of the obtained scintigraphic images and the in formativeness of the results.

Second: Evaluate the scintigraphic result by:

1. Qualitative assessment - according to the colors of the scintigraphic image, corresponding to the degree of accumulation of radiopharmaceuticals (RF). The fixation of radiopharmaceutical in a certain area alerts the nuclear physician to a possible focus of an inflammatory process;

2. Quantitative assessment - calculating the indicator "index of accumulation" (IA). An area of interest $\mathrm{ROI}_{1}$ is determined from the region suspected to be a IP, and a substantially equal second zone located therein into the contralateral region of the body $\left(\mathrm{ROI}_{2}\right)$. Calculating the "index of accumulation" (IA): $\mathrm{ROI}_{1} / \mathrm{ROI}_{2}$. The accumulation index (IA) indicates whether or not there is inflammatory process and what is the intensity of the inflammatory process - weak IA $\leq 1,1$; medium IA $=1,1$ 1,3 ; strong $\geq 1,3$

Third: Assess the sensitivity, specificity and accuracy of leukocyte scintigraphy in the diagnosis of FUO.

Fourth: For each patient, the effective dose $\left(D_{\text {ef }}\right)$ from radiation exposure is assessed by a Swedish doscatolog [8].

First we choose the used radionuclide (99m-Technetium $\left.={ }^{99 \mathrm{~m}} \mathrm{Tc}\right)$; then we selected radiopharmaceuticals $\left({ }^{99 \mathrm{~m}} \mathrm{Tc}-\right.$ hexamethylpropyleneamineoxime $={ }^{99 \mathrm{~m}} \mathrm{Tc}-\mathrm{HMPAO}$ ) and application mode (orally or intravenously); age of the patient (for adults, the dose is up to $15 \mathrm{~g}$ to $10 \mathrm{~g}$ to $7 \mathrm{~g}$ ), and application activity [MBq]

\subsection{Statistical Methods}

Analysis of variance (ANOVA) is a statistical technique for analyzing observation that depends on the effects of one (or more) classification variables (referred as factors) [9]. The analysis is done by partitioning the total variance in the observations into those variations attributable to the different explanatory variables or those attributable to random effects. Each factor or treatment occurs at two or more levels (particular category of a factor). The response variable is influenced not only by the levels of factor but also by external variation.

One-way analysis of variance can be viewed as a special case of bivariate analysis. The variable response variable $X$ is assumed to be continuous random variable and the variable $\mathrm{Y}$ is assumed to be a classification variable with $r$ categories. The interrelationship between the two variables therefore involves a comparison of means. The sampling model is the following: one column of the data matrix contains $N$ observations on a variable $X$, a second column contains observations on a variable $Y$ which classifies the $N$ individuals into $r$ categories (levels) or groups. The $\mathrm{Y}$ variable assumes one of the integer values $1,2, \ldots, r$. The $N$ individuals as a random sample from a population after classification according to $Y$ resulted in totals of $n 1, n 2, \ldots, n r$ observations from the $r$ groups respectively. Equivalently, one could view the sample of size $N$ as the result of selecting random samples of sizes $n 1, n 2, \ldots, n r$. The distribution of $X$ is assumed to be normal for each group. The objective in ANOVA is to use the sample information to test the null hypothesis: the population means of $r$ mutually independent groups are the same .

The total variation in $X$ over the sample is denoted by SST (total sum of squares) and this sum measures the variation in the $X$ values around the grand mean or overall mean. The variation explained by the fitted model is given by SSA, the sum of squares among groups. This sum measures the variation among the group means. For all groups the total within-group sum of squares or Error Sum of squares (SSE) is given by

$$
S S E=S S T-S S A
$$

Under the normality assumption the ratio:

$$
F=(S S A)(N-r) /(S S E)(r-1)
$$

has a $\mathrm{F}$ distribution with $(r-1)$ and $(N-r)$ degree of freedom. For F-test the p-value less than 0,05 was considered statistically significant and null hypothesis is rejected.

When the null hypothesis is rejected, the information that can be obtained is not that the $r$ groups are different from each other. It only provides information that the means of the groups may differ and at least one group may show a difference. This means that it does not provide information on which group differs from which other group. As a result, the comparisons are made with different pairings of groups, undergoing an additional process of verifying which group differs from which other group. This process is referred to as the post-hoc test [9].

If the usual assumption of normality and homogeneity of variance are not satisfied, there are nonparametric alternatives to the F-test for equality of group means. The most commonly used procedure is the Kruskal-Wallis test which used the ranks of the observations [9]. All of the observations are ranked jointly. The rank scores are then assigned to their respective groups. The ranks in each group are summed to yield the rank sums $R i, i=1,2, \ldots, r$. Multiple comparison among groups can be carried out by comparing $(R i-R k)$. Level $\mathrm{p}<0.05$ was considered to indicate a statistically significant differences.

\section{Result and discission}

Calculated all the nuclear medical diagnostic metrics we received:

First: For "efficiency of tagging" (ET) we received $54,46 \%$. The value proves the high quality of the used technique for isolation and labeling of leukocytes, which is a prerequisite for achieving a high quality of the obtained scintigraphic images and high informativeness of the results.

Second: We received "index of accumulation" (IA) $=0$ in 22 patients; IA less 1,1 in 12 patients; IA between 1,1 and 1.3 in 27 patients; and IA more than 1.3 in 13 patients. This means that in 22 patients there is no inflammatory process, in 12 patients there is an inflammatory process but very weak; in 27 patients, the inflammatory process was of a medium intensity, and in 13 patients - it was very intense.

Third: We received 91,07\% sensitivity, 94,44\% specificity and $91,89 \%$ accuracy of leukocyte scintigraphy 
in the diagnosis of FUO. These values are slightly above the published values we are familiar with.

Fourth: The effective dose $\left(\mathrm{D}_{\mathrm{ef}}\right)$ obtained from patients are in the range of $1,72 \mathrm{mSv}$ to $3,44 \mathrm{mSv}$. When comparing the data from the National Center for Radiobiology and Radiation Protection (NCRRP) with the obtained values for $D_{\text {ef }}$ from nuclear methods (NM) we established:

$\mathrm{D}_{\text {ef }}$ Ro $(0,01-1,3 \mathrm{mSv})<\mathrm{D}_{\text {ef }} \mathrm{NM}(1,72 \mathrm{mSv}$ to $3,44 \mathrm{mSv})<$ $\mathrm{D}_{\mathrm{ef}} \mathrm{CT}(2,3-10,0 \mathrm{mSv})<\mathrm{D}_{\text {ef }}$ therapeutic methods

In the study 74 patients are included, aged 7 to 75 years. The mean value \pm standard deviation and $95 \%$ confidence limits (CL) of the measured variables are the following: temperature $(\mathrm{t})-39,1^{0} \mathrm{C} \pm 0,81$ [CL: 38, $9^{0} \mathrm{C}$; $\left.39,3^{0} \mathrm{C}\right]$; leukocytes (le) - 13,09 $\pm 1,74$ [CL: 12,7; 13,5]; ESR - 20,59 $\pm 3,77$ [CL: 19,7; 21,5].

All statistical analyses were conducted using Statistical Package STATISTICA 13.0 [10].

The variable $\mathrm{Y}$ (classification variable) is index of accumulation $(I A)$ which classifies the activity of the inflammatory process in 4 categories:

Without inflammatory process - if $I A=0$ - coded by ' 0 ';

Weak - if $I A<1,1$ - coded by "1";

Medium - if $I A \in[1,1 ; 1,3]$ - coded by "2";

Strong - if $I A>1,3$ - coded by "3".

In the first ANOVA analysis the response variable $X$ is patient's body temperature. For the data of the factor body temperature the ANOVA table is given in Table 1 . The $\mathrm{p}$ value for $F$ statistic of 1,7914 with 3 and 70 degrees of freedom is 0,157 . We conclude, therefore, that there no differences among mean values of the temperature.

TABLE I.

ANOVA results for the factor body temperature

\begin{tabular}{|c|c|c|c|c|c|}
\hline \multirow{2}{*}{$\begin{array}{c}\text { Source of } \\
\text { variation }\end{array}$} & \multicolumn{5}{|c|}{ ANOVA for the body temperature } \\
\cline { 2 - 6 } & $\begin{array}{c}\text { Sum of } \\
\text { Squares }\end{array}$ & $\begin{array}{c}\text { Degree of } \\
\text { Freedom }\end{array}$ & $\begin{array}{c}\text { Mean } \\
\text { Square }\end{array}$ & $\boldsymbol{F}$ & $\boldsymbol{p}$ \\
\hline Among groups & 3.40 & 3 & 1.10 & 1.79 & $>0.05$ \\
\hline Within groups & 44.60 & 70 & 0.60 & & \\
\hline Total & 48.00 & 73 & & & \\
\hline
\end{tabular}

The graph of the means values of temperature and $95 \%$ confidence limits for the four groups of $I A$ are shown on Fig. 1.

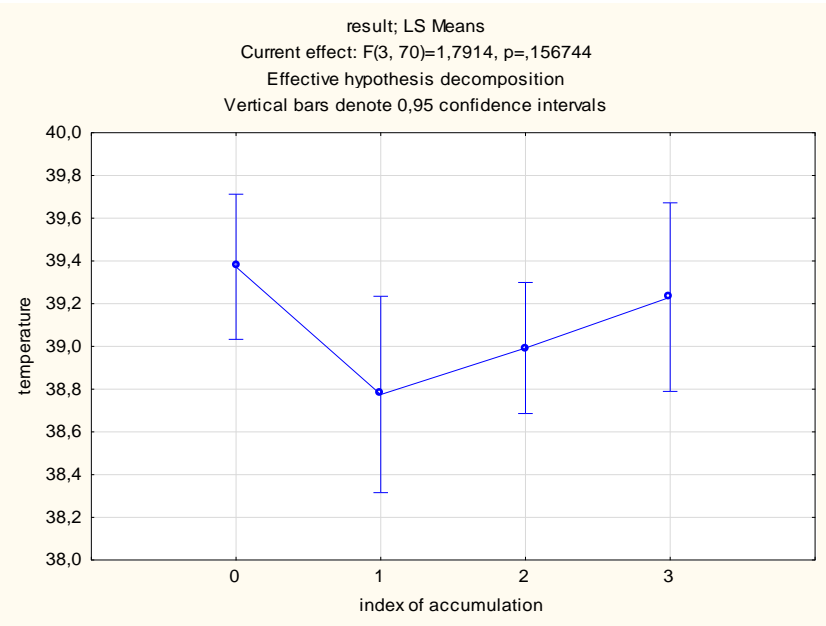

Fig. 1. The mean values and $95 \%$ confidence limits of temperature for the four groups of $I A$.

In the second analysis the response variable $X$ is patient's value of leukocytes. For the data of the factor leukocytes the ANOVA table is given in Table 2. The pvalue for $\mathrm{F}$ statistic is 0,000001 . Therefore, that there are some differences among mean values of the leukocytes.

TABLE II.

ANOVA results for the factor leukocytes

\begin{tabular}{|c|c|c|c|c|c|}
\hline \multirow{2}{*}{$\begin{array}{c}\text { Source of } \\
\text { variation }\end{array}$} & $\begin{array}{c}\text { Sum of } \\
\text { Squares }\end{array}$ & $\begin{array}{c}\text { Degree of } \\
\text { Freedom }\end{array}$ & $\begin{array}{c}\text { Mean } \\
\text { Square }\end{array}$ & $\boldsymbol{F}$ & $\boldsymbol{p}$ \\
\cline { 2 - 6 } & 100.05 & 3 & 33.40 & 19.51 & $<0.05$ \\
\hline Among groups & 119.75 & 70 & 1.71 & & \\
\hline Within groups & 219.80 & 73 & & & \\
\hline Total & & & & & \\
\hline
\end{tabular}

The graph of the mean values of leukocytes and it's 95\% confidence limits for the four groups of $I A$ are shown on Fig. 2.

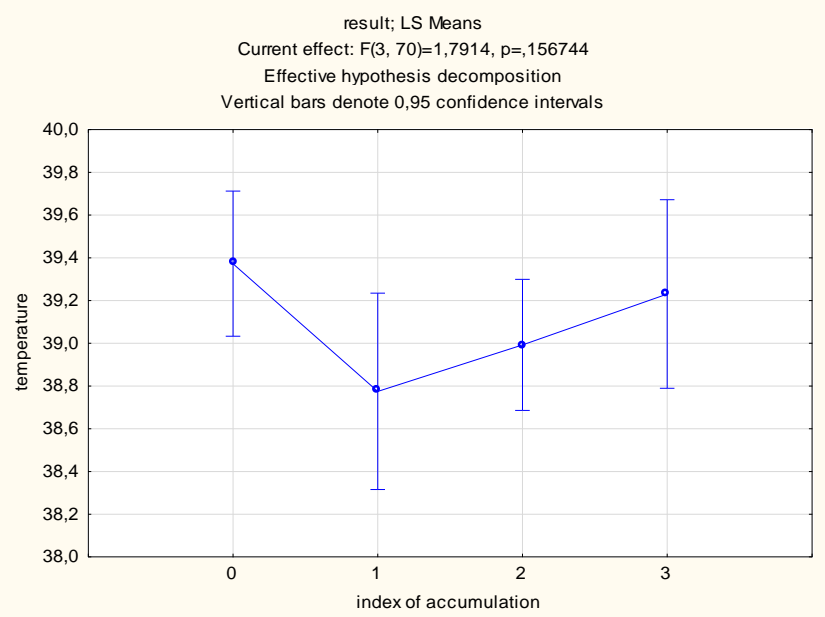

Fig. 1. The mean values and $95 \%$ confidence limits of body temperature for the four groups of $I A$.

Upon examination of the four sample means in Fig. 2 it would appear that the average value of leukocytes is larger 
for strong activity of the inflammatory process than for the other three groups. Received p-levels by Kruskal-Wallis test are given in the Table 4.

The test give us that the mean value of leukocytes of group " 0 " differs from other three groups ( $p$-levels $<0,05)$ and the means of group " 1 "and " 2 " are different of the mean value in group " 3 ".

The last analysis is when the response variable is erythrocyte sedimentation rate. For the data of the factor ESR the ANOVA table is given in Table 3. The p-value for $\mathrm{F}$ statistic is 0,000628 . We conclude, therefore, that there are some differences among mean values of the ESR. The graph of the mean values of erythrocyte sedimentation rate and it's $95 \%$ confidence limits for the four groups of $I A$ are shown on Fig. 3.

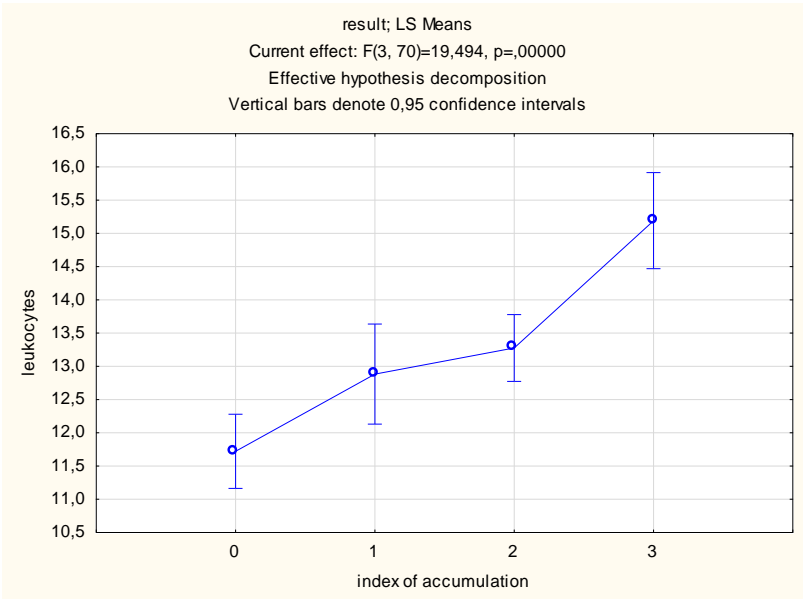

Fig. 2. The mean values and $95 \%$ confidence limits of leukocytes for the four groups of $I A$.

TABLE III.

Post-hoc test (nonparametric ANOVA) for the factor leukocytes.

\begin{tabular}{|c|c|c|c|c|}
\hline \multirow[b]{3}{*}{$\underline{\mathbf{R i}}$} & \multicolumn{4}{|c|}{$\begin{array}{l}\text { Multiple Comparisons p values (2-tailed) for } \\
\text { leukocytes; Independent (grouping) variable: IC } \\
\text { Kruskal-Wallis test: } \mathrm{H}(3, \mathrm{~N}=\mathbf{7 4})=\mathbf{4 0 . 9 6 9 1 8} \\
\mathrm{p}=\mathbf{0 , 0 0 0 0 1}\end{array}$} \\
\hline & $0-R 0:$ & 1-R1: & $2-R 2:$ & $3-R 3:$ \\
\hline & 16,386 & 39 & 41,667 & 63,192 \\
\hline 0 & & 0,0203 & 0,00026 & 0,000001 \\
\hline 1 & 0,020334 & & 1 & 0,02972 \\
\hline 2 & 0,000256 & 1 & & 0,018161 \\
\hline 3 & 0,000001 & 0,0297 & 0,01816 & \\
\hline
\end{tabular}

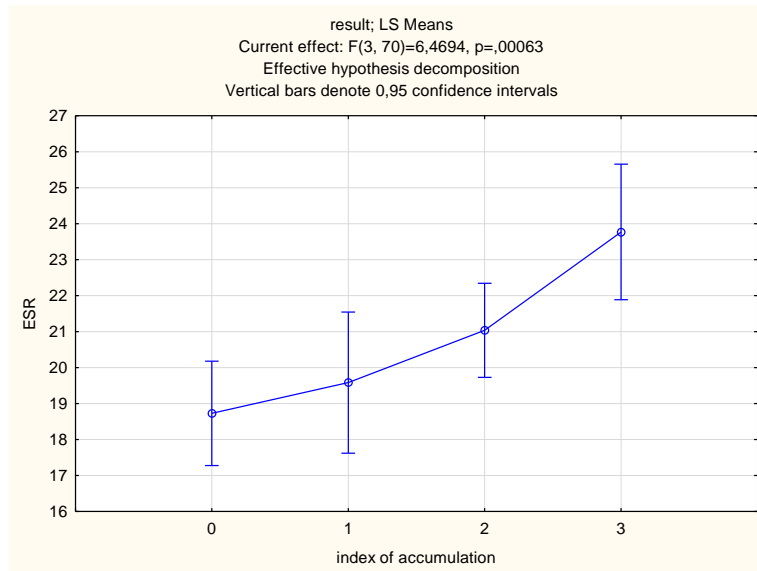

Fig. 3. The mean values and $95 \%$ confidence limits of ESR for the four groups of $I A$.

TABLE IV

ANOVA results for the factor erythrocyte sedimentation rate

\begin{tabular}{|c|c|c|c|c|c|}
\hline \multirow{2}{*}{$\begin{array}{c}\text { Source of } \\
\text { variation }\end{array}$} & $\begin{array}{c}\text { Sum of } \\
\text { Squares }\end{array}$ & $\begin{array}{c}\text { Degree of } \\
\text { Freedom }\end{array}$ & $\begin{array}{c}\text { Mean } \\
\text { Square }\end{array}$ & $\boldsymbol{F}$ & $\boldsymbol{p}$ \\
\hline Among groups & 225.30 & 3 & 75.10 & 6.47 & $<0.05$ \\
\hline Within groups & 812.60 & 70 & 11.60 & & \\
\hline Total & 1037.80 & 73 & & & \\
\hline
\end{tabular}

We conclude from the Kruskal-Wallis test (Table 5) that there is some evidence that group " 3 " yields larger value of erythrocyte sedimentation rate than for the groups coded by "0" (p-level=0,000209) and " $1 "$ (p-level=0,018973). No differences are found between mean values of erythrocyte sedimentation rate of groups " 0 ", "1" and " 2 " (plevels $>0,05)$.

TABLE V.

Post-hoc test (nonparametric ANOVA) for the factor ESR.

\begin{tabular}{|c|c|c|c|c|}
\hline \multirow{2}{*}{} & \multicolumn{5}{|c|}{$\begin{array}{c}\text { Multiple Comparisons p values (2-tailed) for ESR ; } \\
\text { Independent (grouping) variable: } \mathbf{I C} \text { Kruskal-Wallis test: } \\
\mathbf{H}(\mathbf{3}, \mathbf{N}=\mathbf{7 4})=\mathbf{1 8 , 2 3 1 3 9} \mathbf{p}=\mathbf{0 , 0 0 0 4}\end{array}$} \\
\cline { 2 - 5 } & & & $\mathbf{2}-\mathbf{R 2}:$ & $\mathbf{3 - R 3 :}$ \\
\hline \multirow{2}{*}{$\mathbf{R i}$} & 26,432 & 32,167 & 39,222 & 57,577 \\
\hline 0 & & 1,000000 & 0,230304 & 0,000209 \\
\hline 1 & 1,000000 & & 1,000000 & 0,018973 \\
\hline 2 & 0,230304 & 1,000000 & & 0,068785 \\
\hline 3 & 0,000209 & 0,018973 & 0,068785 & \\
\hline
\end{tabular}




\section{Conclusions}

Fever of unknown origin is a life-threatening clinical condition, mainly because of the difficult diagnosis of the cause. Despite the numerous attempts by various specialists in this area, there is still no definite algorithm for diagnosis. In this study, we demonstrated that there is a statistically significant relationship between leukocytes, erythrocyte sedimentation rate (ESR) and the inflammatory process. So we also took another small step on the difficult road to solving the big problem - diagnosis of the fever of unknown origin in Bulgarian.

\section{Acknowledgment}

The authors are fully supported by project DN12/11/20.dec.2017 of Ministry of Education and Science of Bulgaria.

\section{References}

[1] R. G. Petersdorf and P. B. Beeson, "Fever of unexplained origin: report on 100 cases", Medicine, Vol. 40, pp. 1-30, 1961.

[2] E. M. De Kleijn, J. P. Vandenbroucke and J. W. Van der Meer "Fever of unknown origin (FUO). I. A prospective multicenter study of 167 patients with FUO, using fixed epidemiologic entry criteria", Medicine, Vol. 76, pp. 392-400, 1997.

[3] D. C. Knockaert, L. J. Vanneste and H. J. Bobbaers, "Recurrent or episodic fever of unknown origin. Review of 45 cases and survey of the literature", Medicine, Vol. 72, pp. 184-196, 1993.

[4] A. M. Peters and D. Shnier, The imaging of infection and inflammation. London, Kluwer Academic Publishers; 2002 pp.18117.

[5] M. Baymakova, K. Plochev, I. Dikov, G. T. Popov, R. MihaylovaGarnizova, V. Kovaleva and T. Kundurdjiev, "Fever of Unknown Origin in a Bulgarian Hospital: Evaluation of 54 Cases for a Four Year-Period", J Clin Anal Med, Vol. 7(1), pp. 70-75, 2016.

[6] M. Baymakova, G.T. Popov, R. Andonova, V.Kovaleva, I. Dikov and K. Plochev, "Fever of unknown origin and Q-fever: a case series in a Bulgarian hospital", Caspian J Intern Med, Vol. 10 (1), pp. 102-106, 2019.

[7] T. K. Kim, "Understanding one-way ANOVA using conceptual figures", Vol. 70(1), pp. 22-26, 2017.

[8] S. Leide-Svegborn, S. Mattsson, L. Johansson, P. Fernlund and B. Nosslin, "Absorbed Doses to Patients in Nuclear Medicine", Project SSI P1426.04, 2007.

[9] J. D. Jobson, Applied Multivariate Data Analysis, Vol. I, Springer Verlag, 1991, pp. 553-602, 1991.

[10] STATISTICA 13.0, StatSoft Inc, USA.

\section{Creative Commons Attribution License 4.0 (Attribution 4.0 International, CC BY 4.0)}

This article is published under the terms of the Creative Commons Attribution License 4.0

https://creativecommons.org/licenses/by/4.0/deed.en_US 